\title{
Nasal Soft-Tissue Triangle Deformities
}

\author{
Hossam M.T. Foda, MD ${ }^{1}$ \\ ${ }^{1}$ Division of Facial Plastic Surgery, Otolaryngology Department, \\ Alexandria Medical School, Alexandria, Egypt \\ Facial Plast Surg 2016;32:339-344.
}

\begin{abstract}
Address for correspondence Hossam M.T. Foda, MD, Division of Facial Plastic Surgery, Otolaryngology Department, Alexandria Medical School, 11 Roushdy St., Sidi Gaber, Alexandria, Egypt

(e-mail: hfoda@dataxprs.com.eg).
\end{abstract}
Abstract
Keywords
- rhinoplasty
- soft triangle deformities
- revision surgery
- soft triangle graft

The soft-tissue triangle is one of the least areas attended to in rhinoplasty. Any postoperative retraction, notching, or asymmetries of soft triangles can seriously affect the rhinoplasty outcome. A good understanding of the risk factors predisposing to soft triangle deformities is necessary to prevent such problems. The commonest risk factors in our study were the wide vertical domal angle between the lateral and intermediate crura, and the increased length of intermediate crus. Two types of soft triangle grafts were described to prevent and treat soft triangle deformities. The used soft triangle grafts resulted in an excellent long-term aesthetic and functional improvement.
The soft-tissue (ST) triangle is located between the dome of alar cartilage superiorly and the nostril margin inferiorly. The name "soft" triangle came from the lack of cartilage support in this area as it is formed only of skin and soft tissue. The ST triangle is made of two juxtaposed layers of skin, the external nasal skin and the internal vestibular skin, separated by loose areolar tissue and some terminal fibers of nasalis and depressor septi nasi muscles. ${ }^{1,2}$ Aesthetically, the ST triangle should appear as a soft facet that is not too defined or too deep, otherwise it would detract from the aesthetically pleasing tip lobule contour. The shape and appearance of the ST triangle is influenced mainly by the thickness of the lobular skin as well as the strength, shape, and position of alar cartilage. Commonest deformities of the ST triangles include retraction and notching. The soft triangle retraction will form deep pronounced soft triangle facets and can be encountered in both primary as well as revision cases. However, soft triangle notching is encountered mostly in revision cases and may be either isolated or as a part of a wider alar rim notching. The external rhinoplasty approach can result in soft triangle notching if the columellar incision is wrongly placed too high and extending across the nostril apices to join the marginal incision. ${ }^{3,4}$

The aim of this study is to identify the anatomic variations and surgical maneuvers predisposing to soft triangle deformities and to describe soft triangle grafts that can be used to prevent soft triangle retraction and/or notching in noses with high risk of developing such deformities as well as to treat preexisting soft triangle deformities in primary or revision noses.

\section{Patients and Methods}

The study included 150 patients (96 women and 54 men), in which the ST triangle graft was used; 94 (63\%) were revision cases and 56 (37\%) were primary cases.

Evaluation of the surgical outcome of ST triangle grafting depended on clinical examination, comparison of pre- and postoperative photographs, and degree of patients' satisfaction with the aesthetic and functional outcome of procedure.

All cases were operated upon using the external rhinoplasty approach $^{5}$ to allow accurate evaluation of the alar cartilages in their normal anatomic position to detect any specific anatomic factors that may predispose to soft triangle retraction and/or notching. Evaluation included the skin thickness, strength and contour of alar cartilages, orientation of lateral crus, length and inclination of intermediate crus, and horizontal and vertical domal angles between lateral and intermediate crura ( - Fig. 1).

\section{Surgical Considerations}

All cases were operated upon using the external rhinoplasty approach where bilateral marginal incisions are connected via an inverted $\mathrm{V}$-shaped columellar incision. ${ }^{6,7}$ The marginal incision is placed right on the caudal edge of the lateral crus in the outer part, and as we proceed medially, the marginal incision is directed away from the cartilage and toward the alar rim, thus preserving the cuff of vestibular skin caudal to the domal angle, which is the internal lining of the soft triangle (-Fig. 2). Preserving that cuff of skin will facilitate
Issue Theme Challenging Problems in Rhinoplasty; Guest Editor, Hossam M.T. Foda, MD
Copyright (c) 2016 by Thieme Medical Publishers, Inc., 333 Seventh Avenue, New York, NY 10001, USA. Tel: +1(212) 584-4662.
DOI http://dx.doi.org/ 10.1055/s-0036-1585424. ISSN $0736-6825$. 

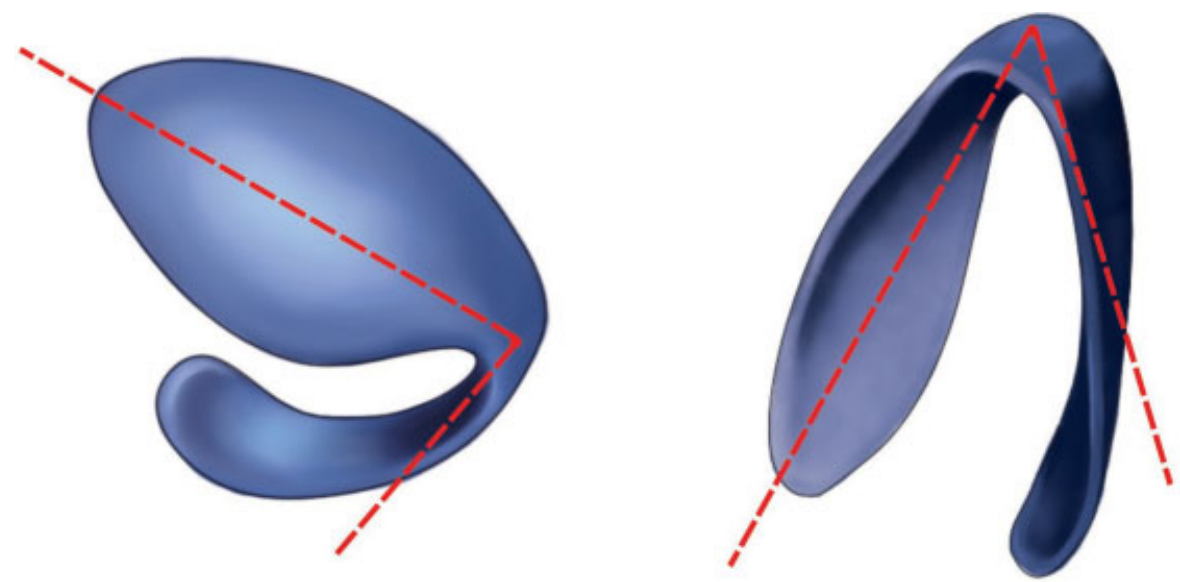

Fig. 1 Right: horizontal domal angle. Left: vertical domal angle between long axis of intermediate and lateral crura.

the subsequent placement and fixation of soft triangle grafts. Evaluation of the soft triangle is done after completing all the surgical maneuvers of rhinoplasty. After the final shape, position, and orientation of tip cartilages have been attained, the nasal skin is redraped to its normal anatomic position, and the lobular skin is squeezed over the tip cartilages for a minimum of 30 seconds followed by inspecting the ST triangles for any retraction or tendency toward retraction, or any degree of notching, or asymmetries.

\section{Soft Triangle Graft}

According to the size and shape of the soft triangle, a 4 to $6 \mathrm{~mm}$ triangular or oval graft is cut out of the excised cephalic parts of lateral crura, which is our preferred cartilage source for the soft triangle graft as it is quite pliable, easily shaped, and holds almost no risk of showing through the skin (-Fig. 3). In revision cases, with soft triangle notching and excessive scar tissue, stronger septal or conchal cartilage was used after fully beveling the edges of the graft to blend with the surrounding tissues. The graft is then secured to the caudal edge of the intermediate and lateral crura with interrupted 6/0 PDS sutures (Polydioxanone, Ethicon Inc., Somerville, $\mathrm{NJ}$ ) in an inverted fashion to bury the knots away from the undersurface of external skin.

\section{Extended Soft Triangle Graft}

In cases where soft triangle retraction is accompanied with alar notching, a triangular graft (4-5 $\mathrm{mm}$ base and 12-15 mm long; - Fig. 4) is fashioned out of strong septal or conchal cartilage, which is carefully thinned out and beveled, and then introduced in a precise pocket, as an alar rim graft, and extended medially, across the soft triangle, until reaching the intermediate crus where it will be fixed to it and to the caudal edge of lateral crus using interrupted $6 / 0$ PDS inverted sutures.

At completion of the procedure, the dorsal skin flap is redraped to its normal anatomic position, and the external rhinoplasty approach incisions are closed ensuring that the marginal incisions are meticulously approximated to avoid any graft exposure and possible infection.
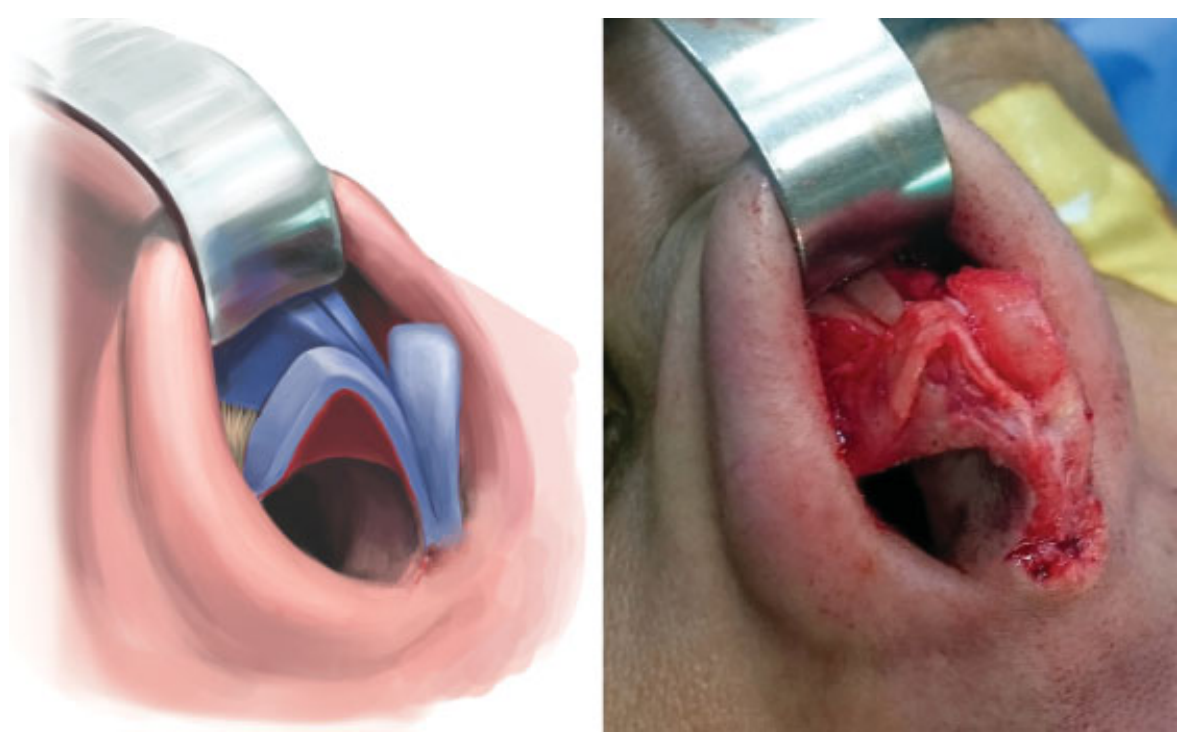

Fig. 2 Diagrammatic illustration and intraoperative photo showing the preservation of the internal lining of the soft-tissue triangle. 


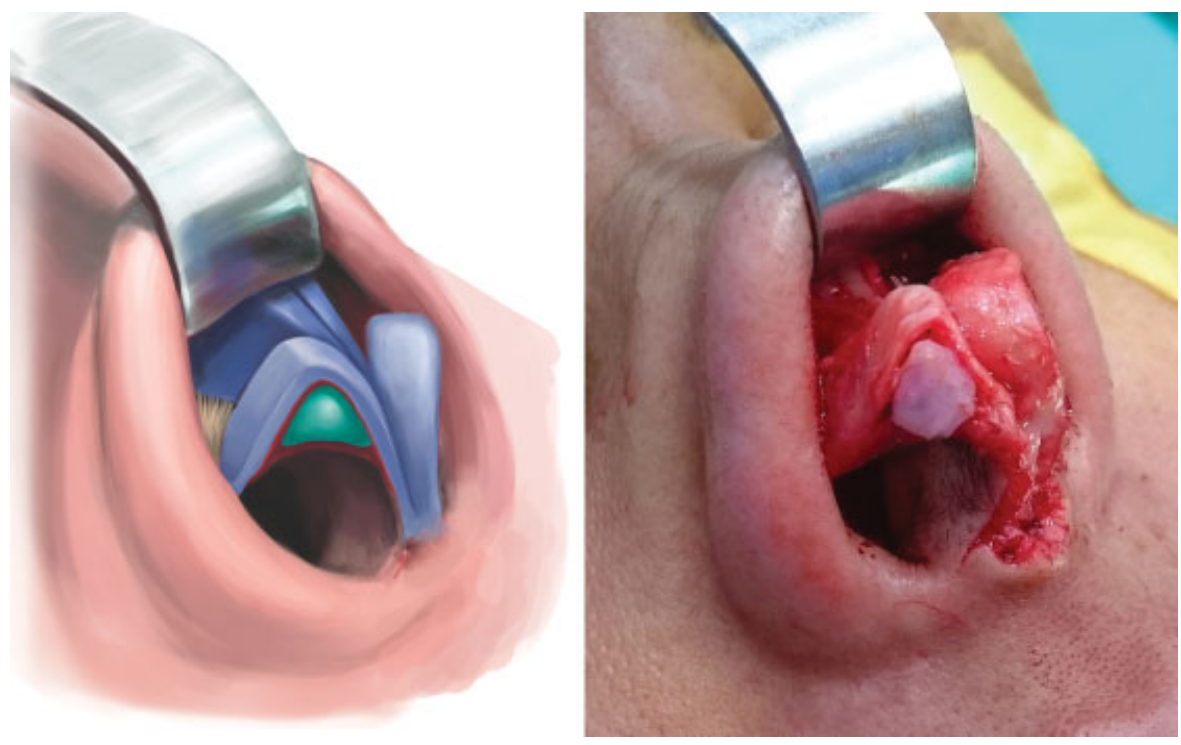

Fig. 3 Diagrammatic illustration and intraoperative photo of the soft-tissue triangle graft.
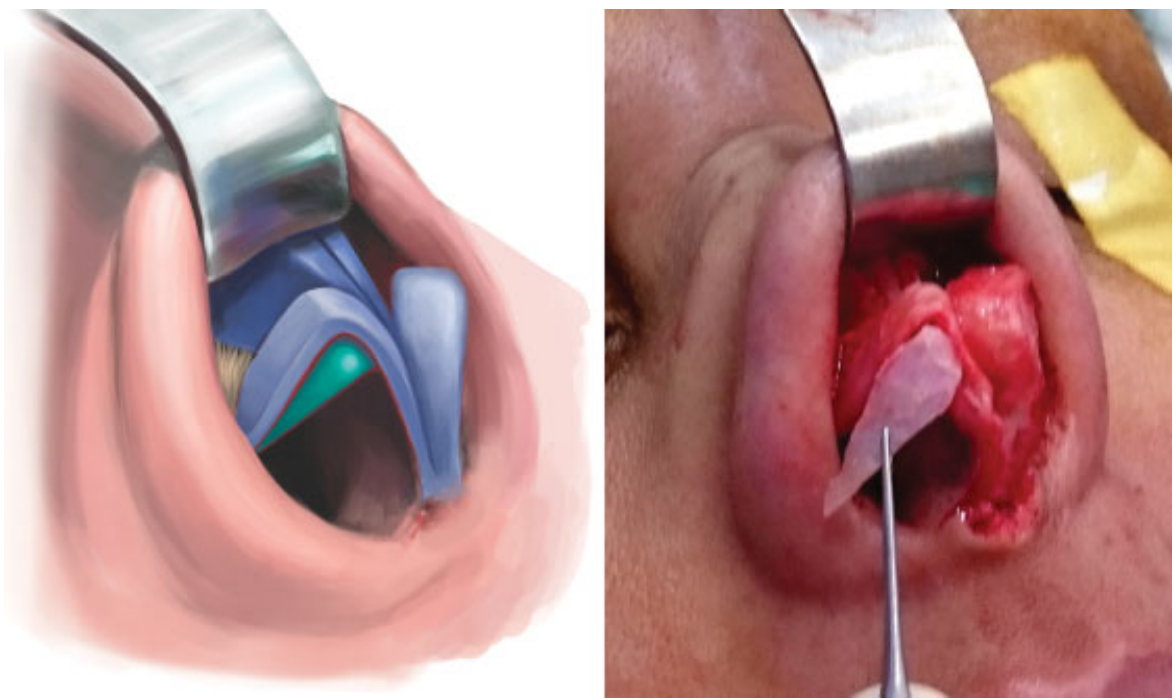

Fig. 4 Diagrammatic illustration and intraoperative photo of the extended soft-tissue triangle graft.

\section{Results}

In 108 (72\%) patients, the soft triangle grafts were used to correct actual soft triangle retraction and/or notching, while in the remaining $42(28 \%)$ patients, they were used prophylactically to guard against possible soft triangle deformities in high-risk cases. The commonest predisposing factors for developing ST deformities in our study was the wide vertical domal angle between the lateral and intermediate crura (-Fig. 1) and the increased length of intermediate crura.

Out of the 150 patients, included in this study, only 122 (81\%) patients were possible to follow-up for more than 1 year after surgery, and their mean period of follow-up was 30 months (range: 1-6 years). The soft triangle grafts resulted in effective long-term correction of ST retraction and notching. No cases of infection, displacement, or extrusion

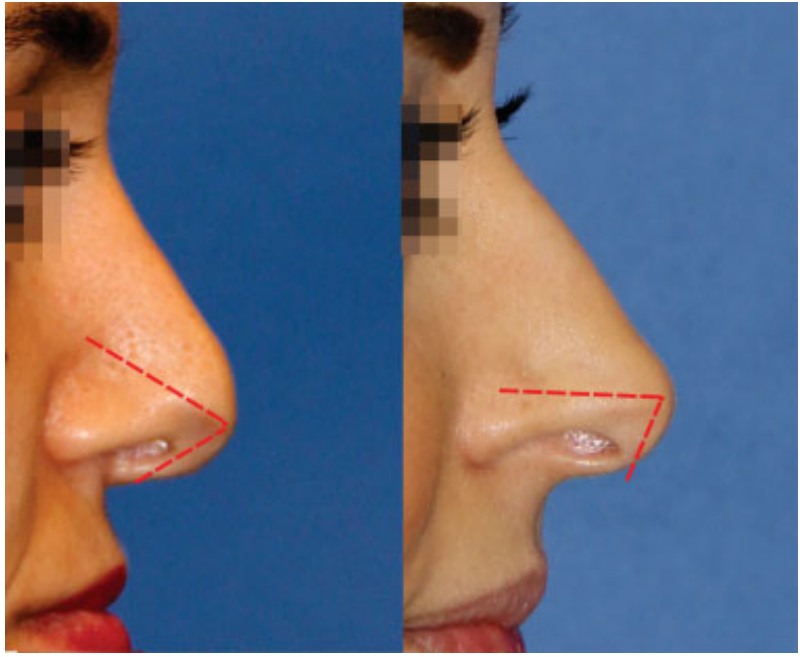

Fig. 5 Wide vertical domal angle due to a malpositioned lateral crus (left) or a hanging intermediate crus (right). 
were encountered. However, revision surgery was required in three cases to correct a sharp visible caudal edge of the graft; those three cases had extended soft triangle grafts made of septal cartilage.

\section{Discussion}

The ST triangle is one of the areas that is usually left unattended in most rhinoplasties. Its appearance is critical to maintaining the natural contour of the nasal tip lobule. Recognizing the patient at risk of developing soft triangle retraction or notching can help implement prophylactic measures to avoid such deformities, thus achieving a satisfying rhinoplasty result. In our

A

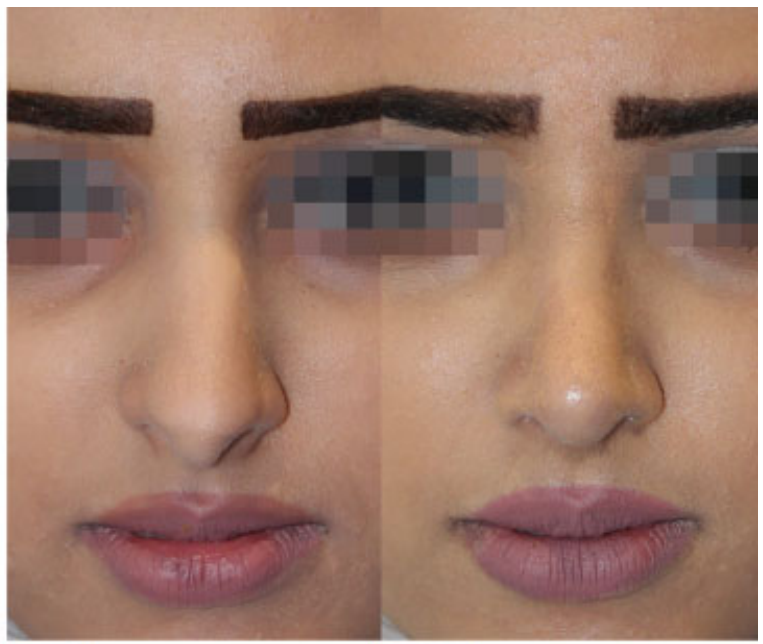

$\mathrm{B}$

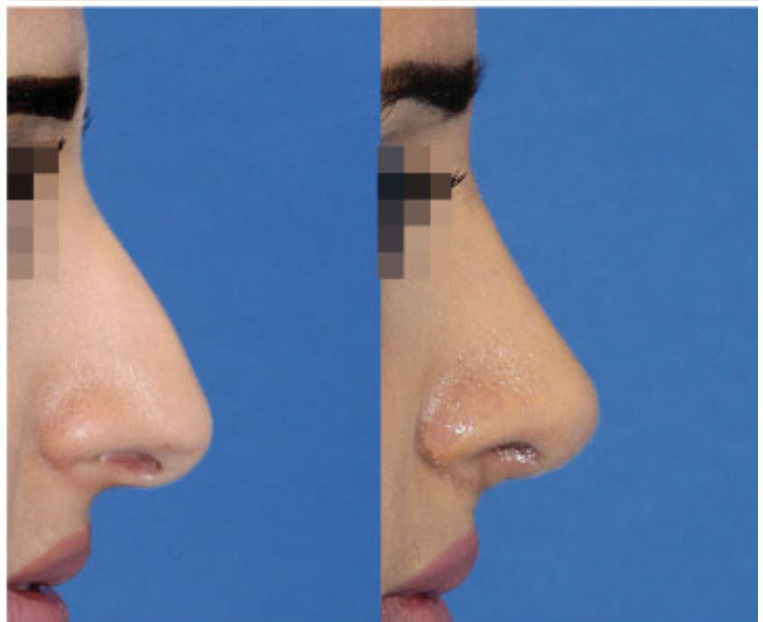

C

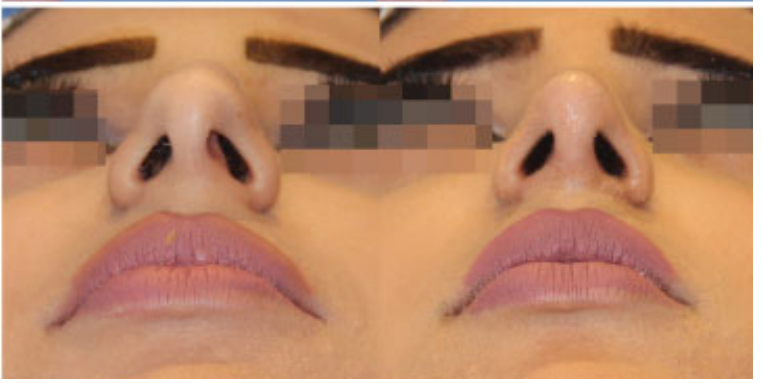

Fig. 6 (A-C) Left: preoperative views of a primary patient with soft triangle retraction. Right: 2-year postoperative views of same patient after using bilateral soft triangle grafts.
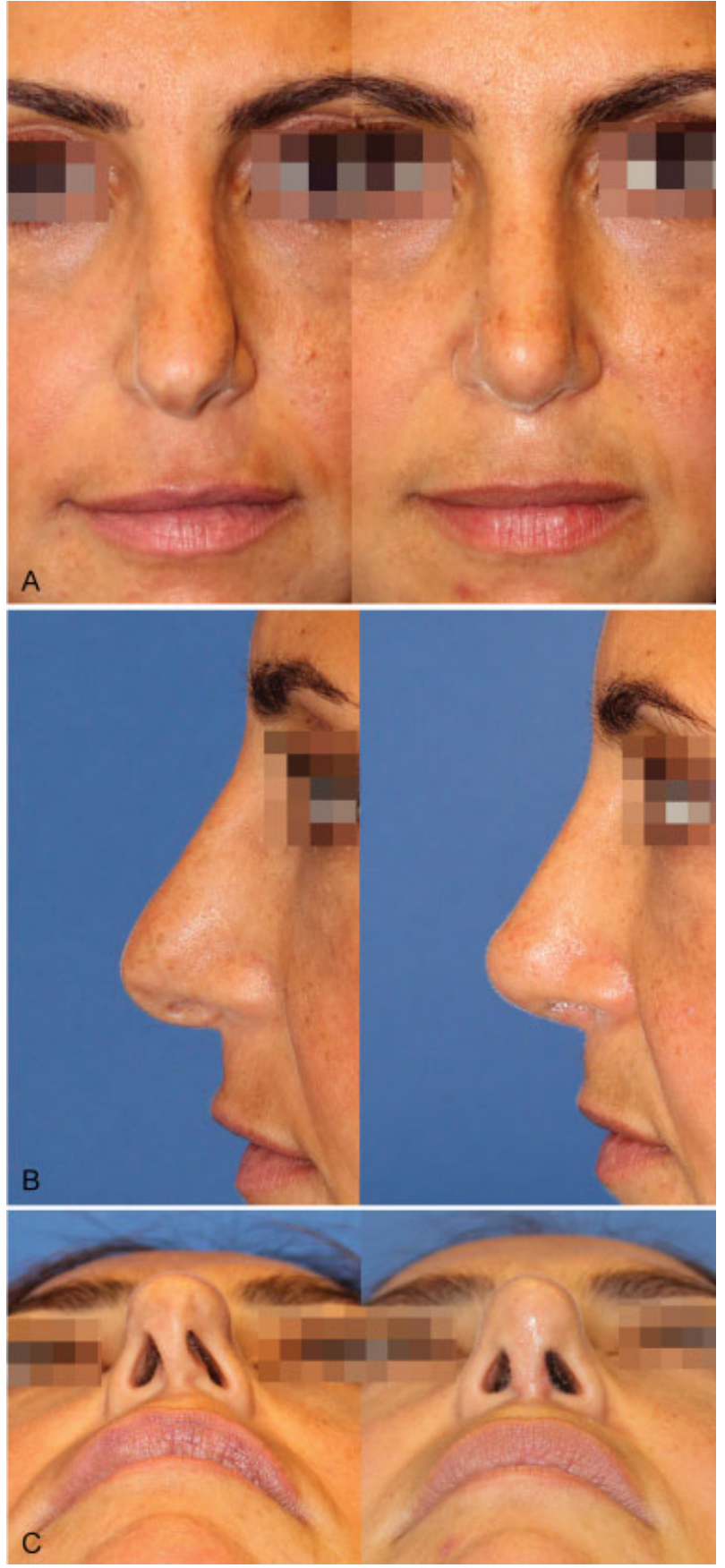

Fig. 7 (A-C) Left: preoperative views of a revision patient with soft triangle retraction. Right: 1-year postoperative views of same patient after using a soft triangle graft on left side.

study, nasal skin thickness was not a significant factor in relation to soft triangle deformities as soft triangle retraction and/or notching was encountered in patients with both thin and thick nasal skin. However, the commonest predisposing factor for soft triangle deformities in our study was the wide vertical domal angle (between the lateral and intermediate crura), which may result from either a malpositioned, vertically oriented, lateral crus, ${ }^{8-10}$ or a hanging intermediate crus and infratip lobule ( widening of the vertical domal angle will stretch the soft triangle skin, thus placing the soft triangle at a high risk of retraction or 

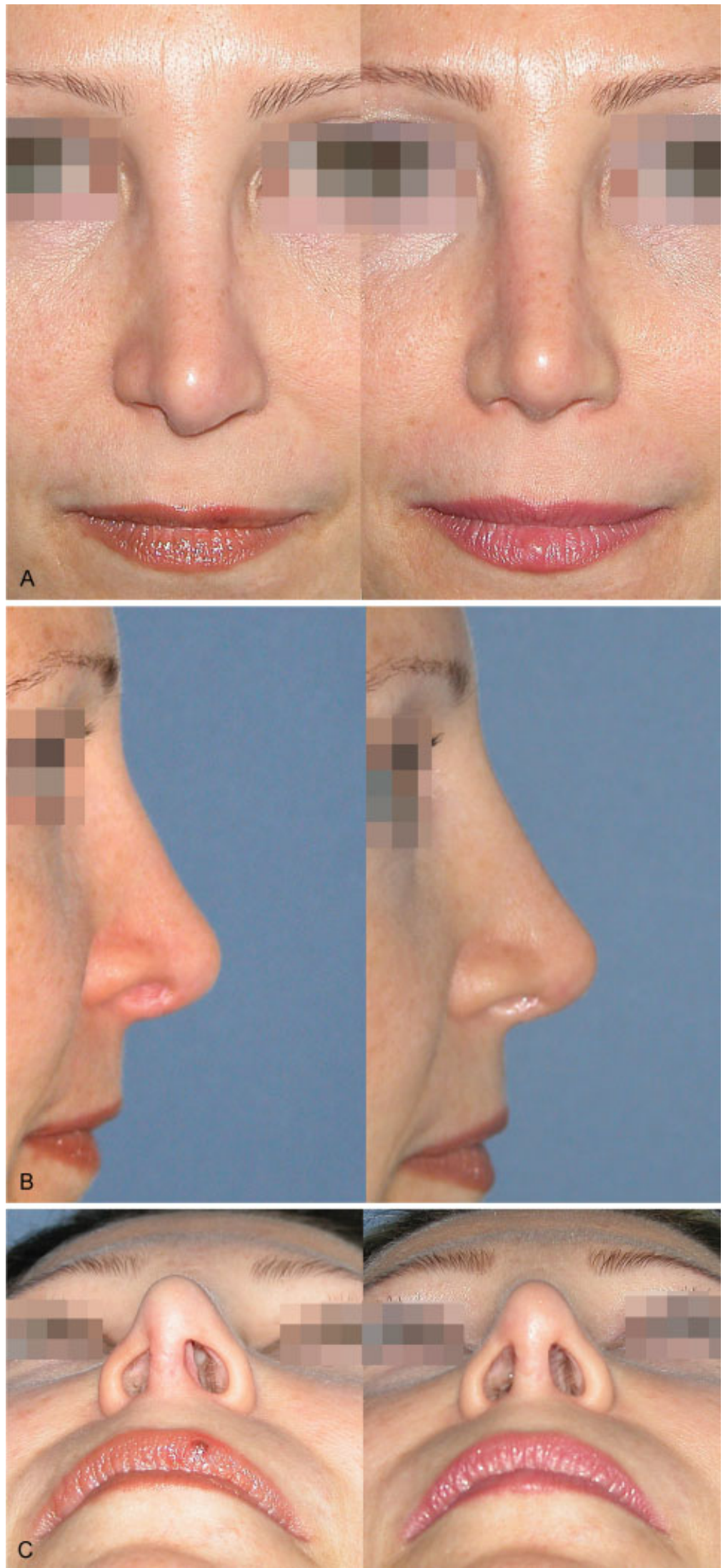

Fig. 8 (A-C) Left: preoperative views of a revision patient with soft triangle retraction and notching. Right: 18-month postoperative views of same patient after using an extended soft triangle graft on right side. 
notching. Another predisposing factor was the long intermediate crus that may be commonly encountered in the Pinocchio nose with overprojected nasal tip or may result from surgical maneuvers that lengthens the intermediate crus, as in the lateral crural steal technique. ${ }^{10-13}$ Accordingly, when using medial crural shortening techniques to deproject nasal tip, it is safer to shorten the intermediate crus and not the medial crus. ${ }^{13-15}$ Also, when performing a large lateral crural steal of more than $4 \mathrm{~mm}$ or performing lateral crural steal in cases with vertically oriented lateral crura, it is helpful to use a soft triangle graft to support the soft triangle skin and prevent the risk of possible retraction.

In the soft triangle grafts used in our study, the cartilage of choice, whenever available, was the cephalic parts of the lateral crura as it is thin and pliable, thus eliminating the risk of the graft edges being visible or palpable. However, in most revision cases with scarring and ST notching, more rigid grafts of septal or conchal cartilage were required to achieve a stable long-lasting correction. On using such rigid grafts, meticulous thinning and beveling of all the graft edges is mandatory to avoid possible graft visibility through the thin soft triangle skin.

The use of soft triangle grafts resulted in effective correction of soft triangle deformities in primary (-Fig. 6) as well as in revision cases ( - Fig. 7). Additionally, the extended soft triangle grafts successfully treated any associated alar rim notching by its downward mobilization of alar rims ( $\mathbf{- F i g . 8}$ ), except in severe cases with deficient vestibular lining that needs to be lengthened by composite grafts. ${ }^{16,17}$ The extended soft triangle grafts can also reverse the functional problems of lateral crural malpositioning ${ }^{18}$ by strengthening the flaccid alae, making them more resistant to inspiratory collapse and thus reducing the need for bulky lateral crural grafts. ${ }^{19,20}$

\section{References}

1 Ali-Salaam P, Kashgarian M, Persing J. The soft triangle revisited. Plast Reconstr Surg 2002;110(1):14-16

2 Nguyen DQA, Motley R, Cooper MA. Repair of nasal soft triangle notching. J Plast Reconstr Aesthet Surg 2008;61(8):966-968
3 Foda HM. Rhinoplasty for the multiply revised nose. Am J Otolaryngol 2005;26(1):28-34

4 Daniel RK. Secondary rhinoplasty following open rhinoplasty. Plast Reconstr Surg 1995;96(7):1539-1546

5 Goodman WS. Recent advances in external rhinoplasty. J Otolaryngol 1981;10(6):433-439

6 Foda HMT. External rhinoplasty: a critical analysis of 500 cases. J Laryngol Otol 2003;117(6):473-477

7 Foda HMT. External rhinoplasty for the Arabian nose: a columellar scar analysis. Aesthetic Plast Surg 2004;28(5):312-316

8 Sheen JH. Aesthetic Rhinoplasty. St. Louis, MO: Mosby; 1978: 432-462

9 Constantian MB. Four common anatomic variants that predispose to unfavorable rhinoplasty results: a study based on 150 consecutive secondary rhinoplasties. Plast Reconstr Surg 2000;105(1): 316-331, discussion 332-333

10 Foda HMT. Management of the droopy tip: a comparison of three alar cartilage-modifying techniques. Plast Reconstr Surg 2003; 112(5):1408-1417, discussion 1418-1421

11 Kridel RW, Konior RJ, Shumrick KA, Wright WK. Advances in nasal tip surgery. The lateral crural steal. Arch Otolaryngol Head Neck Surg 1989;115(10):1206-1212

12 Foda HM, Kridel RW. Lateral crural steal and lateral crural overlay: an objective evaluation. Arch Otolaryngol Head Neck Surg 1999; 125(12):1365-1370

13 Lipsett EM. A new approach surgery of the lower cartilaginous vault. AMA Arch Otolaryngol 1959;70(1):42-47

14 McCurdy JA. Reduction of excessive nasal tip projection with a modified Lipsett technique. Ann Plast Surg 1978;1(5): 478-480

15 Foda HMT. Alar setback technique: a controlled method of nasal tip deprojection. Arch Otolaryngol Head Neck Surg 2001;127(11): 1341-1346

16 Tardy ME Jr, Toriumi D. Alar retraction: composite graft correction. Facial Plast Surg 1989;6(2):101-107

17 Guyuron B. Alar rim deformities. Plast Reconstr Surg 2001;107(3): 856-863

18 Constantian MB. Functional effects of alar cartilage malposition. Ann Plast Surg 1993;30(6):487-499

19 Gunter JP, Friedman RM. Lateral crural strut graft: technique and clinical applications in rhinoplasty. Plast Reconstr Surg 1997; 99(4):943-952, discussion 953-955

20 Toriumi DM, Josen J, Weinberger M, Tardy ME Jr. Use of alar batten grafts for correction of nasal valve collapse. Arch Otolaryngol Head Neck Surg 1997;123(8):802-808 